This is the final peer-reviewed accepted manuscript of:

Detomi, E., M. Morigi, and P. Shumyatsky. "On Bounded Conciseness of Engel-Like Words in Residually Finite Groups." Journal of Algebra, vol. 521, 2019, pp. 1-15.

The final published version is available online at : http://dx.doi.org/10.1016/j.jalgebra.2018.11.020

Rights / License:

The terms and conditions for the reuse of this version of the manuscript are specified in the publishing policy. For all terms of use and more information see the publisher's website. 


\title{
On bounded conciseness of Engel-like words in residually finite groups
}

\author{
Eloisa Detomi, Marta Morigi, and Pavel Shumyatsky
}

\begin{abstract}
Given a group-word $w$ and a group $G$, the verbal subgroup $w(G)$ is the one generated by all $w$-values in $G$. The word $w$ is said to be boundedly concise if for each positive integer $m$ there exists a number depending only on $m$ and $w$ bounding the order of $w(G)$ whenever the set of $w$-values in a group $G$ has size at most $m$. In the present article we show that various generalizations of the Engel word are boundedly concise in residually finite groups.
\end{abstract}

\section{Introduction}

Given a group-word $w=w\left(x_{1}, \ldots, x_{k}\right)$, the verbal subgroup $w(G)$ of a group $G$ determined by $w$ is the subgroup generated by the set $G_{w}$ consisting of all values $w\left(g_{1}, \ldots, g_{k}\right)$, where $g_{1}, \ldots, g_{k}$ are elements of $G$. A word $w$ is said to be concise if whenever $G_{w}$ is finite for a group $G$, it always follows that $w(G)$ is finite. More generally, a word $w$ is said to be concise in a class of groups $\mathcal{X}$ if whenever $G_{w}$ is finite for a group $G \in \mathcal{X}$, it always follows that $w(G)$ is finite. In the sixties Hall raised the problem whether all words are concise. In $1989 \mathrm{~S}$. Ivanov [17] (see also [24, p. 439]) solved the problem in the negative. On the other hand, the problem for residually finite groups remains open (cf. Segal [27, p. 15] or Jaikin-Zapirain [18]). In recent years some limited progress with respect to this problem was made. In particular, it was shown in $[\mathbf{2}]$ that if $w$ is a multilinear commutator word and $n$ is a prime-power, then the word $w^{n}$ is concise in the class of residually finite groups. Further examples of words that are concise in residually finite groups were discovered in [13]. The work [5] deals with conciseness of words of Engel type.

Set $\left[x,{ }_{0} y\right]=x,\left[x,{ }_{1} y\right]=[x, y]=x^{-1} y^{-1} x y$ and $\left[x,{ }_{i+1} y\right]=\left[\left[x,{ }_{i} y\right], y\right]$ for $i \geq 1$. The word $\left[x,{ }_{n} y\right]$ is called the $n$th Engel word. Due to $[\mathbf{8}]$ and, independently, $[\mathbf{1}]$ we know that the $n$th Engel word is concise whenever $n \leq 4$. It is still unknown whether the $n$th Engel word is concise in the case where $n \geq 5$. In [5] it was shown, among other things, that the Engel words are concise in residually finite groups. More generally, it was shown that words implying virtual nilpotency are boundedly concise in residually finite groups. A word $w$ is said to imply virtual nilpotency if every finitely generated metabelian group where $w$ is a law has a nilpotent subgroup of finite index. Such words admit several important characterizations (see $[\mathbf{3}, \mathbf{4}$,

2010 Mathematics Subject Classification. Primary 20E26, 20F45, 20F10.

Key words and phrases. Words, Conciseness. 
10]). It follows from a theorem of Gruenberg [11] that the Engel words imply virtual nilpotency.

Recall that a word $w$ is boundedly concise in a class of groups $\mathcal{X}$ if for every integer $m$ there exists a number $\nu=\nu(\mathcal{X}, w, m)$ such that whenever $\left|G_{w}\right| \leq m$ for a group $G \in \mathcal{X}$ it always follows that $|w(G)| \leq \nu$. In $[7]$ it is shown that every word which is concise in the class of all groups is actually boundedly concise. There is a conjecture that every word which is concise in residually finite groups is boundedly concise (cf. [9]) but this probably will remain open for some time.

In the present article we will prove the following result.

THEOREM 1.1. The word $\left[\left[x_{1}^{q}, x_{2}^{q}\right],{ }_{n}\left[x_{3}, x_{4}\right]\right]$ is boundedly concise in residually finite groups for any nonnegative integers $n, q$.

Note that the word $\left[\left[x^{q}, y^{q}\right],{ }_{n} z\right]$ implies virtual nilpotency so in a sense the word $\left[\left[x_{1}^{q}, x_{2}^{q}\right],{ }_{n}\left[x_{3}, x_{4}\right]\right]$ is a minimal word for which conciseness was unknown. The word $\left[x^{q}, y^{q}\right]^{n}$ remains a mystery even when $q=n$ is a prime.

We also deal with weakly rational words. Recall that a word $w$ is weakly rational if for every finite group $G$ and for every integer $e$ relatively prime to $|G|$, the set of $w$-values in $G$ is closed under taking eth powers of its elements. Examples of such words include the words $\left[\ldots\left[x_{1}^{n_{1}}, x_{2}\right]^{n_{2}}, \ldots, x_{k}\right]^{n_{k}}$ for any integers $n_{1}, n_{2}, \ldots, n_{k}$ (see [13]). In particular, the lower central words $\gamma_{k}=\left[x_{1}, x_{2}, \ldots, x_{k}\right]$ are weakly rational. Quite possibly, all multilinear commutator words are weakly rational but this remains an open question.

It is not difficult to see that weakly rational words are boundedly concise in residually finite groups. In this article we will establish that whenever $v=$ $v\left(x_{1}, \ldots, x_{k}\right)$ is a weakly rational word and $n$ is a nonnegative integer the word $\left[v,_{n} y\right]$ is boundedly concise in residually finite groups. In fact, we will establish more general results. Let us say that a word $w$ is commutator-closed if the set of $w$-values in any group is closed under taking commutators of its elements. Note that in particular the lower central words $\gamma_{k}$ are commutator-closed.

THEOREM 1.2. Let $k, n, q$ be nonnegative integers and $u$ be the word $y^{q}$, or the word $\left[y_{1}, y_{2}\right]^{q}$, or any commutator-closed word. Suppose that $v=v\left(x_{1}, \ldots, x_{k}\right)$ is a weakly rational word all of whose values in any group are also $u^{-1}$-values. Then the word $\left[v,{ }_{n} u\right]$ is boundedly concise in residually finite groups.

The above theorem guarantees conciseness in residually finite groups of many words, in particular of the word $\left[v,{ }_{n} y\right]$, where $v=v\left(x_{1}, \ldots, x_{k}\right)$ is a weakly rational word. Further, if $v$ is a commutator or a $q$ th power, Theorem 1.2 implies bounded conciseness in residually finite groups of the words $\left[v,{ }_{n}\left[y_{1}, y_{2}\right]\right]$ and $\left[v,_{n} y^{q}\right]$, respectively. Other obvious examples of words whose conciseness in residually finite groups follows from Theorem 1.2 are the words of the form $\left[v,_{n} u\right]$, where $v=\left[x_{1}^{q}, x_{2}, \ldots, x_{k}\right]$ and $u=\gamma_{l}$ for $l \leq k$.

\section{Sufficient conditions for nilpotency of a group}

Recall that an element $x$ of a group $G$ is called a (left) Engel element if for any $g \in G$ there exists a positive integer $n=n(x, g)$ such that $\left[g,_{n} x\right]=1$. If $n$ can be chosen independently of $g$, then $x$ is a (left) $n$-Engel element. Here and throughout the article we use the expression " $(a, b, \ldots)$-bounded" to mean that a quantity is bounded by a certain number depending only on the parameters $a, b, \ldots$.

In this section we will establish the following two propositions. 
Proposition 2.1. Let $k, n, q, s, t$ be positive integers and $w$ a word. Let $G$ be a residually finite group satisfying the identity $w \equiv 1$ and generated by elements $a_{1}, \ldots, a_{k}$ such that

$$
[a_{i}, \underbrace{\left[x_{1}, x_{2}\right]^{q}, \ldots,\left[x_{1}, x_{2}\right]^{q}}_{n},\left[x_{1}, x_{2}\right]^{s}]=1
$$

for each $x_{1}, x_{2} \in G$ and each $i=1, \ldots, k$. Assume further that the elements $a_{1}^{-1}, \ldots, a_{k}^{-1}$ are $t$-Engel. Then $G$ is nilpotent with $(k, n, q, s, t, w)$-bounded class.

We say that a word $u$ is commutator-closed if in any group $G$ the set $G_{u}$ is closed under taking commutators of its elements. Moreover, given two words $v$ and $w$ we write

$$
v \preceq w
$$

if and only if $G_{v} \subseteq G_{w}$ for every group $G$.

Proposition 2.2. Let $k, n, q, s, t$ be positive integers, and let $u, v$ be words such that $u$ is commutator-closed and $v^{-1} \preceq u$. Let $G$ be a residually finite group satisfying the identity $[v, \underbrace{u^{q}, \ldots, u^{q}}_{n}, u^{s}] \equiv 1$ and assume that all $v^{-1}$-values are $t$ Engel in $G$. Suppose that $H$ is a subgroup of $G$ generated by $k$ v-values. Then $H$ is nilpotent with $(k, n, q, s, t, u, v)$-bounded class.

The proof uses Lie methods in the spirit of Zelmanov's solution of the Restricted Burnside Problem, which states that, given two positive integers $m$ and $n$, the order of an $m$-generated finite group of exponent $n$ is bounded by a number depending only on $m$ and $n$. For the reader's convenience we collect some definitions and facts on Lie algebras associated with groups (see [28] or [30] for further information).

Let $L$ be a Lie algebra over a field. We use the left normed notation; thus if $l_{1}, \ldots, l_{n}$ are elements of $L$ then

$$
\left[l_{1}, \ldots, l_{n}\right]=\left[\ldots\left[\left[l_{1}, l_{2}\right], l_{3}\right], \ldots, l_{n}\right] .
$$

An element $y \in L$ is called ad-nilpotent if $a d y$ is nilpotent, i.e. there exists a positive integer $n$ such that $\left[x,{ }_{n} y\right]=0$ for all $x \in L$. If $n$ is the least integer with the above property then we say that $y$ is ad-nilpotent of index $n$. Let $X$ be any subset of $L$. By a commutator in elements of $X$ we mean any element of $L$ that could be obtained from elements of $X$ by repeated operation of commutation with an arbitrary system of brackets, including the elements of $X$. Here the elements of $X$ are viewed as commutators of weight 1 . Denote by $F$ the free Lie algebra over the same field as $L$ on countably many free generators $x_{1}, x_{2}, \ldots$ Let $f=f\left(x_{1}, \ldots, x_{n}\right)$ be a non-zero element of $F$. The algebra $L$ is said to satisfy the identity $f \equiv 0$ if $f\left(a_{1}, \ldots, a_{n}\right)=0$ for any $a_{1}, \ldots, a_{n} \in L$. In this case we say that $L$ is $P I$. We are now in position to quote a theorem of Zelmanov $[\mathbf{3 0}, \mathbf{3 1}]$ which has numerous important applications to group theory. A detailed proof of this result recently appeared in $[\mathbf{3 2}]$.

THEOREM 2.3. Let $L$ be a Lie algebra generated by finitely many elements $a_{1}, a_{2}, \ldots, a_{m}$ such that all commutators in $a_{1}, a_{2}, \ldots, a_{m}$ are ad-nilpotent. If $L$ is $P I$, then it is nilpotent.

The following lemma provides a sufficient condition for an element of a Lie algebra to be ad-nilpotent. 
Lemma 2.4. [21, Lemma 5] Let $p$ be a prime and $k$ a positive integer. Assume that $L$ is a Lie algebra over a field of characteristic $p$ generated by $a_{1}, \ldots, a_{m}$. If $y \in L$ is an element such that $\left[a_{i}, p^{k} y\right]=0$ for $i=1, \ldots, m$, then $y$ is ad-nilpotent of index at most $p^{k}$.

Let $G$ be a group. Given a prime $p$, a Lie algebra can be associated with the group $G$ as follows. We denote by $D_{i}=D_{i}(G)$ the $i$ th dimension subgroup of $G$ in characteristic $p$ (see for example [15, Chap. 8]). These subgroups form a central series of $G$ known as the Zassenhaus-Jennings-Lazard series. Set $L(G)=$ $\oplus D_{i} / D_{i+1}$. Then $L(G)$ can naturally be viewed as a Lie algebra over the field $\mathbb{F}_{p}$ with $p$ elements. For an element $x \in D_{i} \backslash D_{i+1}$ we denote by $\tilde{x}$ the element $x D_{i+1} \in L(G)$.

Lemma 2.5 (Lazard, [20]). For any $x \in G$ we have $(\operatorname{ad} \tilde{x})^{p}=\operatorname{ad}\left(\tilde{x^{p}}\right)$.

The next lemma provides a criterion for a Lie algebra to be $P I$.

Lemma 2.6 (Wilson, Zelmanov, [29]). Let $G$ be any group satisfying a group law. Then $L(G)$ is $P I$.

Let $L_{p}(G)$ be the subalgebra of $L(G)$ generated by $D_{1} / D_{2}$. Often, important information about the group $G$ can be deduced from nilpotency of the Lie algebra $L_{p}(G)$. In particular, we have the following theorem. Recall that a group $G$ is said to be residually- $p$ if for each nontrivial $x \in G$ there exists a normal subgroup $N$ of finite $p$-power index such that $x \notin N$. By a linear group we understand a subgroup of $G L(r, K)$ for some field $K$ and a positive integer $r$.

THEOREM 2.7. If $G$ is a finitely generated residually-p group such that $L_{p}(G)$ is nilpotent, then $G$ is a linear group.

A proof of the above theorem can be obtained as follows. Suppose that $G$ can be generated with $m$ elements and $L_{p}(G)$ is nilpotent of class $c$. Let $Q$ be a finite homomorphic image of $p$-power order of $G$. Obviously, $Q$ can be generated with $m$ elements and $L_{p}(Q)$ is nilpotent of class at most $c$. By $[\mathbf{1 9}$, Proposition $1]$, the group $Q$ has a powerful characteristic subgroup of $(p, c, m)$-bounded index. It follows that the rank of $Q$ is $(p, c, m)$-bounded (see $[6])$. Since this happens for each finite homomorphic image of $p$-power order of $G$, we conclude that $G$ has a $p$-congruence system of finite rank. By a well-known theorem of Lubotzky (see $[\mathbf{6}$, Theorem B6]) the group $G$ is linear.

The following result is Lemma 3.5 in $[\mathbf{2 8}]$.

LEMMA 2.8. Let $G$ be a finitely generated residually finite-nilpotent group. For each prime $p$ let $J_{p}$ be the intersection of all normal subgroups of $G$ of finite p-power index. If $G / J_{p}$ is nilpotent for each $p$ then $G$ is nilpotent.

LEMma 2.9. Suppose that $G$ is a residually finite group such that $L_{p}(G)$ is nilpotent for each prime $p$. Assume additionally that $G$ is generated by finitely many Engel elements. Then $G$ is nilpotent.

Proof. By Lemma 2.8 it is sufficient to prove that $G / J_{p}$ is nilpotent for any prime $p$. The algebra $L_{p}(G)$ is isomorphic to $L_{p}\left(G / J_{p}\right)$. Theorem 2.7 shows that $G / J_{p}$ is linear while a result of Gruenberg [12] states that a linear group generated by Engel elements is locally nilpotent. Therefore, $G / J_{p}$ is nilpotent.

Lemma 2.10. Let $G$ be a group generated by Engel elements. 
(1) If $G$ is finite, then it is nilpotent.

(2) If $G$ is soluble, then it is locally nilpotent.

Proof. The first statement is well-known Baer's theorem ([14, Satz III.6.15]). The second statement is due to Gruenberg [11].

Now we are ready to prove Proposition 2.1 and Proposition 2.2.

Proof of Proposition 2.1. Since the group $G$ is generated by the Engel elements $a_{1}^{-1}, \ldots, a_{k}^{-1}$, it is a residually finite-nilpotent group (see Lemma 2.10). We prove first that $G$ is nilpotent and then show that the nilpotency class of $G$ is $(k, n, q, s, t, w)$-bounded. To establish that $G$ is nilpotent, by Lemma 2.9 , it is enough to prove that $L_{p}(G)$ is nilpotent for each prime $p$.

Let us choose a prime $p$ and set $L=L_{p}(G)$. By Lemma 2.6, $L$ is $P I$. Write $\tilde{a}_{1}, \ldots, \tilde{a}_{k}$ for the generators of $L$ corresponding to the generators $a_{1}, \ldots, a_{k}$ of $G$, and let $q_{1}$ and $s_{1}$ be the maximum $p$-powers dividing $q$ and $s$, respectively. Since we have $\left[a_{i},{ }_{n} y^{q}, y^{s}\right]=1$ for each commutator $y=\left[g_{1}, g_{2}\right] \in G$ and each $i=1, \ldots, k$, the Lazard lemma (Lemma 2.5) shows that in the Lie algebra we have

$$
\left[\tilde{a}_{i},{ }_{\left(q_{1} n+s_{1}\right)} b\right]=0,
$$

whenever $b$ is a commutator of weight at least two in the generators $\tilde{a}_{i}$. Let $p^{l}$ be the least $p$-power such that $q_{1} n+s_{1} \leq p^{l}$. We deduce from Lemma 2.4 that every commutator of weight at least two in the generators $\tilde{a}_{i}$ is ad-nilpotent with index at most $p^{l}$. Further, the generators $\tilde{a}_{i}$ are ad-nilpotent with index at most $t$ in $L$, because the elements $a_{1}^{-1}, \ldots, a_{k}^{-1}$ are $t$-Engel in $G$. Therefore, by Zelmanov's theorem (Theorem 2.3), $L$ is nilpotent. Taking into account Lemma 2.9 we conclude that $G$ is nilpotent, as we wanted to prove.

It remains to show that the nilpotency class of $G$ is $(k, n, q, s, t, w)$-bounded. Suppose that this is false. Then there exists an infinite sequence $\left(G_{i}\right)_{i>1}$ of nilpotent groups satisfying the hypotheses of the proposition such that the nilpotency class of $G_{i}$ tends to infinity as $i$ does. In each group $G_{i}$ we choose $k$ generators $a_{i 1}, \ldots, a_{i k}$ satisfying the hypotheses of the proposition. Here the elements $a_{i 1}, \ldots, a_{i k}$ are not necessarily pairwise distinct. Let $C$ be the Cartesian product of the groups $G_{i}$ and let $y_{1}, \ldots, y_{k}$ be the elements of $C$ such that the $i$-th component of $y_{j}$ is equal to $a_{i j}$ for every $i \geq 1$ and $1 \leq j \leq k$. Obviously, each of the elements $y_{j}^{-1}$ is $t$-Engel in $C$. Moreover, $\left[y_{j},{ }_{n}\left[x_{1}, x_{2}\right]^{q},\left[x_{1}, x_{2}\right]^{s}\right]=1$ for each $x_{1}, x_{2} \in C$ and each $i=1, \ldots, k$.

Let $H$ be the subgroup of $C$ generated by $y_{1}, \ldots, y_{k}$. Since every group $G_{i}$ is a homomorphic image of $H$, the subgroup $H$ is not nilpotent. On the other hand, being a finitely generated residually nilpotent group, $H$ is residually finite. Thus, by what we proved above, $H$ is nilpotent. This is a contradiction.

In what follows we write $\langle X\rangle$ to denote the subgroup generated by a set $X$.

Proof of Proposition 2.2. We argue as in the proof of Proposition 2.1. Let $H$ be a subgroup of $G$ generated by $k v$-values $a_{1}, \ldots, a_{k}$. Since $a_{1}^{-1}, \ldots, a_{k}^{-1}$ are Engel elements, $H$ is a residually finite-nilpotent group (see Lemma 2.10). We prove first that $H$ is nilpotent and then show that the nilpotency class of $H$ is $(k, n, s, q, t, u, v)$-bounded.

To establish that $H$ is nilpotent, by Lemma 2.9 it is sufficient to show that $L_{p}(H)$ is nilpotent for each prime $p$. Thus, we choose a prime $p$ and set $L=L_{p}(H)$. Note that, by Lemma 2.6, $L$ is $P I$. 
Write $\tilde{a}_{1}, \ldots, \tilde{a}_{k}$ for the generators of $L$ corresponding to the generators $a_{1}, \ldots$, $a_{k}$ of $H$, and let $q_{1}$ and $s_{1}$ be the maximum $p$-powers dividing $q$ and $s$, respectively. Since we have $\left[a_{i},{ }_{n} y^{q}, y^{s}\right]=1$ for each $u$-value $y \in G_{u}$ and each $i=1, \ldots, k$, the Lazard lemma (Lemma 2.5) shows that in the Lie algebra we have

$$
\left[\tilde{a}_{i},\left(q_{1} n+s_{1}\right) \tilde{y}\right]=0 .
$$

In particular, since the word $u$ is commutator-closed and the elements $a_{i}^{-1}$ are $u$-values, we also have that

$$
\left[\tilde{a}_{i},\left(q_{1} n+s_{1}\right) b\right]=0
$$

whenever $b$ is a commutator in the generators $\tilde{a}_{i}$. Let $p^{l}$ be the least $p$-power such that $q_{1} n+s_{1} \leq p^{l}$. We deduce from Lemma 2.4 that every commutator in the generators $\tilde{a}_{i}$ is ad-nilpotent with index at most $p^{l}$. Therefore, by Zelmanov's theorem (Theorem 2.3), $L$ is nilpotent. So, by Lemma 2.9, we conclude that $H$ is nilpotent, as we wanted to prove.

It remains to show that the nilpotency class of $H$ is $(k, n, q, s, t, u, v)$-bounded. Suppose that this is false. Then there exists an infinite sequence $\left(G_{i}\right)_{i \geq 1}$ of groups satisfying the hypotheses of the proposition and in each group $G_{i}$ there exist $k v$ values $a_{i 1}, \ldots, a_{i k}$ such that the nilpotency class of the subgroups $H_{i}=\left\langle a_{i 1}, \ldots, a_{i k}\right\rangle$ tends to infinity as $i$ does. Here the elements $a_{i 1}, \ldots, a_{i k}$ are not necessarily pairwise distinct. Let $C$ be the Cartesian product of the groups $G_{i}$ and let $y_{1}, \ldots, y_{k}$ be the elements of $C$ such that the $i$-th component of $y_{j}$ is equal to $a_{i j}$ for every $i \geq 1$ and $1 \leq j \leq k$. Note that all $v^{-1}$-values are $t$-Engel in $C$. Moreover, $\left[y_{j},{ }_{n} z^{q}, z^{s}\right]=1$ for each $u$-value $z \in C_{u}$ and each $i=1, \ldots, k$.

Let $D$ be the subgroup of $C$ generated by $y_{1}, \ldots, y_{k}$. Since every group $H_{i}$ is a homomorphic image of $D$, the subgroup $D$ is not nilpotent. On the other hand, being a finitely generated residually nilpotent group, $D$ is residually finite.

Thus, by what we proved above, $D$ is nilpotent. This yields the desired contradiction.

\section{Useful lemmas}

In this section we gather preliminary results which will be needed for the proofs of the main theorems.

LEMma 3.1. Let $G$ be a finite group and $w$ a word such that $\left|G_{w}\right| \leq m$.

(1) There exists an $m$-bounded integer s such that $G^{s} \leq C_{G}(w(G))$.

(2) The order of the commutator subgroup $w(G)^{\prime}$ is m-bounded.

ProOf. The first statement follows from the fact that the group $G / C_{G}(w(G))$ acts on the finite set $G_{w}$ by conjugation and hence it embeds in $S_{m}$. In particular, the order of $w(G) / Z(w(G))$ is at most $m$ ! and thus the second statement follows from Shur's Theorem (see [25, p. 102]).

Next lemma is Exercise 3.11 in [16]. It establishes that the commutator $\left[x_{1}, x_{2}\right]$ is a weakly rational word.

LEMma 3.2. Let $G$ be a finite group and $t$ a commutator in $G$. Then each generator of the cyclic subgroup $\langle t\rangle$ is a commutator.

In the following lemmas we collect some observations and results from [5]. 
Lemma 3.3. Let $G$ be a group. Assume that $A$ is a normal abelian subgroup of $G$ and let $t \in G$. Then $\left[a b,{ }_{n} t\right]=\left[a,{ }_{n} t\right]\left[b,{ }_{n} t\right]$ for every $a, b \in A$ and for every $n \geq 1$.

LEMMA 3.4. [5, Lemma 9] Let $G=U\langle t\rangle$ be a group that is a product of a normal subgroup $U$ and a cyclic subgroup $\langle t\rangle$. Assume that $U$ is nilpotent of class $c$ and there exists a generating set $A$ of $U$ such that $\left[a,{ }_{n} t\right]=1$ for every $a \in A$. Then $G$ is nilpotent of $(c, n)$-bounded class.

LEMMA 3.5. Let $w=w\left(x_{1}, \ldots, x_{k}\right)$ be a word and $n$ a positive integer. There is a word $\eta$ in $k(n+1)$ variables such that

$$
\left[w,{ }_{n} y\right]=\eta\left(x_{1}, x_{2}, \ldots, x_{k}, x_{1}^{y}, x_{2}^{y}, \ldots, x_{k}^{y}, \ldots, x_{1}^{y^{n}}, x_{2}^{y^{n}}, \ldots, x_{k}^{y^{n}}\right) .
$$

By definition, a group-word is a nontrivial element of the free group $F=$ $F\left(x_{1}, x_{2}, \ldots\right)$ of countable rank. Let $F_{i}$ denote the $i$ th term of the lower central series of $F$. We say that a word $w$ has degree $j$ if $w \in F_{j}$ and $w \notin F_{j+1}$.

The next lemma is of central importance in our arguments.

Lemma 3.6. [5, Lemma 10] Let $w=w\left(x_{1}, x_{2}, \ldots, x_{k}\right)$ be a word. Let $G$ be a nilpotent group of class c generated by $k$ elements $a_{1}, a_{2}, \ldots, a_{k}$ and let $p$ be a prime. Denote by $X$ the set of all conjugates in $G$ of elements of the form $w\left(a_{1}^{i}, a_{2}^{i}, \ldots, a_{k}^{i}\right)$, where $i$ ranges over the set of all integers not divisible by $p$ and assume that $|X| \leq m$ for some integer $m$. Then $|\langle X\rangle|$ is $(c, m)$-bounded.

Recall that, given two words $v$ and $w$, we write $v \preceq w$ if and only if $G_{v} \subseteq G_{w}$ for every group $G$.

LEMMA 3.7. Let $w=\left[v,_{n} u\right]$ where $v$ and $u$ are words such that $v^{-1} \preceq u$. Let $G$ be a finite group such that $\left|G_{w}\right| \leq m$. Then $G$ has a normal subgroup $T$ of $m$-bounded order such that in $G / T$ the $v^{-1}$-values are $(2 n+2)$-Engel.

Proof. Using Lemma 3.1 we may assume that $w(G)$ is abelian.

Let $y$ be a $v$-value and let $x$ be any element of $G$. By the usual commutator formulae we have $\left[x, y^{-1}, y^{-1}\right]=\left[y^{x y^{-1}}, y^{-1}\right]$. Therefore, since $y$ is a $v$-value and $y^{-1}$ is a $u$-value,

$$
\left[x,{ }_{(n+1)} y^{-1}\right]=\left[y^{x y^{-1}},{ }_{n} y^{-1}\right]
$$

is a $w$-value.

Since $w(G)$ is abelian, whenever $z \in w(G)$ and $i$ is an integer, we have

$$
\left[z^{i},{ }_{(n+1)} y^{-1}\right]=\left[z,{ }_{(n+1)} y^{-1}\right]^{i} .
$$

Moreover, by the above argument, $\left[z^{i},{ }_{(n+1)} y^{-1}\right]$ is a $w$-value. Thus $\left[z,_{(n+1)} y^{-1}\right]^{i}$ is a $w$-value for each integer $i$. As $\left|G_{w}\right| \leq m$, it follows that the elements of the form $\left[z,{ }_{(n+1)} y^{-1}\right]$, with $z \in w(G)$ and $y \in \bar{G}_{v}$, have order at most $m$.

Using that $w(G)$ an abelian group of rank at most $m$ we observe that the elements of $w(G)$ of order dividing $m$ ! generate a normal subgroup of $G$ of $m$ bounded order. Passing to the quotient over this subgroup we may assume that $\left[z,{ }_{(n+1)} y^{-1}\right]=1$ for all $z \in w(G)$. On the other hand, $\left[x,_{(n+1)} y^{-1}\right] \in w(G)$ for all $x \in G$. Hence, $\left[x,_{(n+1)} y^{-1},{ }_{(n+1)} y^{-1}\right]=1$ and $y^{-1}$ is $(2 n+2)$-Engel in $G$.

The following two results are corollaries of Propositions 2.1 and 2.2 respectively, and they are among the key steps in the proofs of the main theorems. 
COROLlary 3.8. Let $u$ be either the word $y^{q}$ or the word $\left[y_{1}, y_{2}\right]^{q}$, where $q$ is a positive integer, and let $v$ be a word such that $v^{-1} \preceq u$. Consider the word $w=\left[v,{ }_{n} u\right]$, where $n$ is a positive integer. Let $G$ be a finite group with at most $m$ values of the word $w$. Then $G$ has a normal subgroup $N$ of $(w, m)$-bounded order, such that, for every integer $k$, in $G / N$ any $k$ v-values generate a nilpotent subgroup of $(w, m, k)$-bounded class.

Proof. By Lemma 3.7, since $v^{-1} \preceq u$, there exists a normal subgroup $T$ of $m$-bounded order such that all $v^{-1}$-values in $G / T$ are $(2 n+2)$-Engel. Passing to the quotient over $T$ we assume, without loss of generality, that all $v^{-1}$-values are $(2 n+2)$-Engel in $G$.

Let $K$ be the subgroup generated by $k v$-values $a_{1}, \ldots, a_{k}$. For each $x_{1}, x_{2} \in K$ the element $\left[a_{i},{ }_{n}\left[x_{1}, x_{2}\right]^{q}\right]$ is a $w$-value. We know from Lemma 3.1 that $G^{s} \leq$ $C_{G}(w(G))$ for some $m$-bounded integer $s$. Therefore

$$
\left[a_{i, n}\left[x_{1}, x_{2}\right]^{q},\left[x_{1}, x_{2}\right]^{s}\right]=1,
$$

for every $i$ and $x_{1}, x_{2} \in K$. Since the elements $a_{1}^{-1}, \ldots, a_{k}^{-1}$ are $(2 n+2)$-Engel, we deduce from Proposition 2.1 that $K$ is nilpotent of $(w, m, k)$-bounded class.

COROLlary 3.9. Let $u$ be a commutator-closed word and let $v$ be a word such that $v^{-1} \preceq u$. Consider the word $w=\left[v,{ }_{n} u\right]$, where $n$ is a positive integer. Let $G$ be a finite group with at most $m$ values of the word $w$. Then $G$ has a normal subgroup $N$ of $(w, m)$-bounded order, such that, for every integer $k$, in $G / N$ any $k$ $v$-values generate a nilpotent subgroup of $(w, m, k)$-bounded class.

Proof. By Lemma 3.7, since $v^{-1} \preceq u$, there exists a normal subgroup $T$ of bounded order such that in $G / T$ all $v^{-1}$-values are $(2 n+2)$-Engel. Passing to the quotient over $T$ we assume, without loss of generality, that all $v^{-1}$-values are $(2 n+2)$-Engel in $G$.

We know that $G^{s} \leq C_{G}(w(G))$ for some $m$-bounded integer $s$. For each $v$-value $a$ and $u$-value $z$, the element $\left[a,_{n} z\right]$ is a $w$-value. Hence

$$
\left[a_{n} z, z^{s}\right]=1 \text {, }
$$

and $G$ satisfies the identity

$$
\left[v,{ }_{n} u, u^{s}\right] \equiv 1 .
$$

By Proposition 2.2 we conclude that any $k v$-values generate a nilpotent subgroup of $(w, m, k)$-bounded class.

\section{Proofs of the theorems}

Now we are ready to prove our main results.

Proof of Theorem 1.2. Let $w=\left[v,{ }_{n} u\right]$, where $v$ is a weakly rational word and $u$ is the word $y^{q}$, or the word $\left[y_{1}, y_{2}\right]^{q}$, or any commutator-closed word such that $v^{-1} \preceq u$.

We need to show that if $G$ is a residually finite group with at most $m$ values of the word $w$, then the order of $W=w(G)$ is $(w, m)$-bounded. Evidently, it suffices to establish this result for finite quotients of $G$. Therefore without loss of generality we assume that $G$ is finite.

Because of Lemma 3.1 we may pass to the quotient $G / W^{\prime}$ and assume that $W$ is abelian. If $u$ is the word $y^{q}$ or the word $\left[y_{1}, y_{2}\right]^{q}$, we apply Corollary 3.8. If $u$ is a commutator-closed word, we apply Corollary 3.9. In any case, $G$ has a normal 
subgroup $N$ of $(w, m)$-bounded order such that, for every integer $k$, in $G / N$ any $k v$-values generate a nilpotent subgroup of $(w, m, k)$-bounded class. Passing to the quotient over $N$ we assume, without loss of generality, that any $k v$-values in $G$ generate a nilpotent subgroup of $(w, m, k)$-bounded class. In particular, $v(G)$ is nilpotent.

Now fix a $w$-value $\left[g{ }_{n} t\right]$ with $g \in G_{v}$ and $t \in G_{u}$. We use Lemma 3.5 and write $\left[g,{ }_{n} t\right]=\eta\left(g, g^{t}, \ldots, g^{t^{n}}\right)$ for an appropriate word $\eta=\eta\left(x_{1}, x_{2}, \ldots, x_{n+1}\right)$. Set $H=\left\langle g, g^{t}, \ldots, g^{t^{n}}\right\rangle$. Being generated by $n+1 v$-values, $H$ is nilpotent of $(w, m)$-bounded nilpotency class.

Assume first that $v(G)$ is a $p$-group for a prime $p$. Then $H$ is a $p$-group as well. Since the word $v$ is weakly rational, $g^{i} \in G_{v}$ for every integer $i$ coprime to $p$.

Since

$$
\eta\left(g^{i}, g^{i t}, \ldots, g^{i t^{n}}\right)=\left[g^{i},{ }_{n} t\right],
$$

it follows that $\eta\left(g^{i}, g^{i t}, \ldots, g^{i t^{n}}\right) \in G_{w}$ for every integer $i$ coprime to $p$. In particular the set

$$
X=\left\{\eta\left(g^{i}, g^{i t}, \ldots, g^{i t^{n}}\right)^{x} \mid x \in H,(i, p)=1\right\}
$$

is a subset of $G_{w}$ and hence $|X| \leq m$.

Since the nilpotency class of $H$ is $(w, m)$-bounded, we deduce from Lemma 3.6 that the order of the element $\eta\left(g, g^{t}, \ldots, g^{t^{n}}\right)$ is $(w, m)$-bounded. Thus, the order of an arbitrary $w$-value $\left[g,{ }_{n} t\right]$ is bounded by a number which depends only on $w$ and $m$. Since $W$ is an abelian subgroup generated by $m$ such elements, we conclude that the order of $W$ is $(w, m)$-bounded. Thus, in the particular case where $v(G)$ is a $p$-group, the proposition is proved. It is important to note that we proved the existence of a bound, say $B$, for $|W|$, which does not depend on $p$.

We will now deal with the case where $v(G)$ is not necessarily a $p$-group. Let $p_{1}, \ldots, p_{s}$ be the set of prime divisors of the order of $v(G)$. Recall that $v(G)$ is nilpotent and so any Hall subgroup of $v(G)$ is normal in $G$. For each $i=1, \ldots, s$ let $N_{i}$ denote the Hall $p_{i}{ }^{\prime}$-subgroup of $v(G)$. The result obtained in the case where $v(G)$ is a $p$-group implies that for any $i$ the image of $W$ in $G / N_{i}$ has order at most $B$. It follows that $W$ embeds into a direct product of abelian groups of order at most $B$. Therefore the exponent of $W$ divides $B$ !. Thus $W$ is an abelian group with $m$ generators and exponent dividing $B$ !. We conclude that the order of $W$ is $(w, m)$-bounded, as required.

Proof of Theorem 1.1. Set $w=\left[\left[x_{1}^{q}, x_{2}^{q}\right],{ }_{n}\left[x_{3}, x_{4}\right]\right]$ and $v=\left[x_{1}^{q}, x_{2}^{q}\right]$. It is sufficient to prove that whenever $G$ is a finite group in which the set of $w$-values is finite of order $m$ then the order of $W=w(G)$ is bounded in terms of $w$ and $m$.

Since $w$ depends on 4 variables and $\left|G_{w}\right| \leq m$, the group $G$ contains a subgroup $T$ with at most $4 m$ generators such that $w(T)=w(G)$. Therefore without loss of generality we can assume that $G$ is a finite group generated by at most $4 m$ elements. Moreover, by Lemma 3.1, we can also assume that $W$ is abelian of rank at most $m$.

We will repeatedly use the fact that, for any positive integer $s$, the order of the subgroup of $W$ generated by all elements of order at most $s$ is $(s, m)$-bounded since $W$ is an abelian $m$-generated group. The proof will be split in several steps.

STEP 1. Note that $v$ is a commutator. Thus we can apply Corollary 3.8, and conclude that $G$ has a normal subgroup $N$ of $(w, m)$-bounded order, such that, for every integer $k$, in $G / N$ any $k v$-values generate a nilpotent subgroup of $(w, m, k)$ bounded class. Passing to the quotient over $N$ we assume, without loss of generality, 
that any $k v$-values in $G$ generate a nilpotent subgroup of $(w, m, k)$-bounded class.

STEP 2. Let $H=G^{q}$ be the subgroup generated by all $q$ th powers in $G$. By the solution of the Restricted Burnside Problem, the index of $H$ in $G$ is $(m, q)$ bounded and hence $H$ is generated by an $(m, q)$-bounded number of elements. The main result in [23] states that each element of $H$ is a product of $(m, q)$-boundedly many $q$ th powers. By Proposition 1.2 in [22], each element of $H^{\prime}$ is a product of $m$-boundedly many commutators of the form $\left[h_{1}, h_{2}\right]$. Writing both $h_{1}$ and $h_{2}$ as a product of $(m, q)$-boundedly many $q$ th powers and using the usual commutator identities we see that each commutator $\left[h_{1}, h_{2}\right]$, where $h_{1}, h_{2} \in H$, is a product of $(m, q)$-boundedly many $v$-values (recall that $v=\left[x^{q}, y^{q}\right]$ ). So, for any $a_{1}, a_{2} \in H^{\prime}$ there exist $(m, q)$-boundedly many $v$-values that generate a subgroup containing both $a_{1}, a_{2}$.

In view of what we proved in STEP 1, the subgroup $\left\langle a_{1}, a_{2}\right\rangle$ is nilpotent of $(w, m)$-bounded class $r$, in particular $\left[a_{1}, r a_{2}\right]=1$. It follows that $H^{\prime}$ is $r$ Engel. Since the $r$ th Engel word implies virtual nilpotency, a theorem of Burns and Medvedev [4, Theorem A] implies that $H^{\prime}$ has a characteristic subgroup $E$ of $(w, m)$-bounded exponent such that $H^{\prime} / E$ has $(w, m)$-bounded nilpotency class. As $E \cap W$ has $(w, m)$-bounded order, we can pass to the quotient $G / E$ and assume that $H^{\prime}$ has $(w, m)$-bounded nilpotency class.

STEP 3. We argue by induction on the nilpotency class $c$ of $H^{\prime}$. The case $H^{\prime}=1$ is trivial so we assume that $c \geq 2$. Let $M$ be the last term of the lower central series of $H^{\prime}$. By induction, the image of $W$ in $G / M$ is of bounded order.

We will show that $G$ contains a normal subgroup of $(w, m)$-bounded order such that, for any arbitrary commutator $a \in G$ the image of $H^{\prime}\langle a\rangle$ in the factor group is nilpotent with $(w, m)$-bounded class.

Let $a$ be a commutator and $b$ a $q$ th power. Since $M$ is abelian, by Lemma 3.3 the element

$$
\left[[x, b],{ }_{n} a\right]^{q^{i}}=\left[\left[x^{q^{i}}, b\right],{ }_{n} a\right]
$$

is a $w$-value for every $x \in M$ and for every integer $i$. As $\left|G_{w}\right| \leq m$, we deduce that the exponent of the subgroup $\left[[M, b],{ }_{n} a\right]$ is bounded by an integer $s$ depending only on $m$ and $q$. Note also that $s$ does not depend on $a$. Passing to the quotient over the subgroup generated by all elements of $W$ of order at most $s$ !, we can assume that

$$
\left[[M, b],{ }_{n} a\right]=1
$$

for all $q$ th powers $b$. It follows from Lemma 3.3 that $\left[[M, H],{ }_{n} a\right]=1$.

Let us consider the quotient group $\bar{G}=G /[M, H]$. Clearly, $\bar{M} \leq Z(\bar{H})$ (here the bar notation has the natural meaning). Remark that $M$ is generated by commutators in elements of $H$. If $\left[\bar{h}_{1}, \bar{h}_{2}\right] \in \bar{M}$, with $h_{1}, h_{2} \in H$, then by the standard commutator identities and the fact that $\bar{M} \leq Z(\bar{H})$ we have $\left[\bar{h}_{1}, \bar{h}_{2}\right]^{i}=\left[\bar{h}_{1}^{i}, \bar{h}_{2}\right]=$ $\left[\bar{h}_{1}, \bar{h}_{2}^{i}\right]$ for every integer $i$. Thus

$$
\left[\left[\bar{h}_{1}, \bar{h}_{2}\right],{ }_{n} \bar{a}\right]^{q^{2 i}}=\left[\left[\bar{h}_{1}, \bar{h}_{2}\right]^{q^{2 i}},{ }_{n} \bar{a}\right]=\left[\left[\bar{h}_{1}^{q^{i}}, \bar{h}_{2}^{q^{i}}\right]_{n} \bar{a}\right]
$$

is a $w$-value for every integer $i$. As $\left|G_{w}\right| \leq m$, it follows that $\left[\left[\bar{h}_{1}, \bar{h}_{2}\right],{ }_{n} \bar{a}\right]$ has $(w, m)$ bounded order. We therefore deduce that $\left[\bar{M},{ }_{n} \bar{a}\right]$ has $(w, m)$-bounded exponent.

Back in the group $G$, we will show that $W$ has an $a$-invariant series

$$
W=W_{0} \geq W_{1} \geq W_{2} \geq W_{3} \geq 1
$$


such that the factors $W_{0} / W_{1}$ and $W_{2} / W_{3}$ are of $(w, m)$-bounded order and $a$ acts as an $n$-Engel automorphism on $W_{1} / W_{2}$ and $W_{3}$.

Indeed, we set

$$
W=W_{0}, \quad W_{1}=W \cap M, \quad W_{2}=\left[W_{1},{ }_{n} a\right], \quad W_{3}=W \cap[M, H] .
$$

Since $W M / M$ has $(w, m)$-bounded order, it follows that $W_{0} / W_{1}$ has $(w, m)$-bounded order as well. As $W$ is abelian of rank at most $m$ and the subgroup $\bar{W}_{2}=$ $\left[\bar{W}_{1},{ }_{n} \bar{a}\right] \leq\left[\bar{M},{ }_{n} \bar{a}\right]$ has $(w, m)$-bounded exponent in the quotient group $G /[M, H]$, we deduce that $W_{2} / W_{3}$ has $(w, m)$-bounded order. It is self-evident that $a$ acts as an $n$-Engel automorphism on $W_{1} / W_{2}$. Finally, since $\left[[M, H],{ }_{n} a\right]=1$, we observe that $\left[W_{3},{ }_{n} a\right]=1$.

We know from Lemma 3.1 that there exists an $m$-bounded positive integer $j$ such that $a^{j}$ centralizes $W$. Let $V_{1}=W_{2}\langle a\rangle$. Note that $W_{3}\left\langle a^{j}\right\rangle \leq Z_{n}\left(V_{1}\right)$, since $\left[W_{3},{ }_{n} a\right]=1$. Here $Z_{i}(K)$ denotes the $i$ th term of the upper central series of a group $K$. Thus the index of $Z_{n}\left(V_{1}\right)$ in $V_{1}$ is $(w, m)$-bounded. Therefore, by a version of Baer's theorem (see the proof of 14.5.1 in [26]), $\gamma_{n+1}\left(V_{1}\right)$ has $(w, m)$-bounded order $r$. We may pass to the quotient over the subgroup generated by all elements of $W$ of order at most $r$ and assume that $\gamma_{n+1}\left(V_{1}\right)=1$. Thus, $a$ acts as an $n$-Engel automorphism on $W_{2}$. Hence, it acts as a (2n)-Engel automorphism on $W_{1}$.

Let $V=W\langle a\rangle$. We see that $W_{1}\left\langle a^{j}\right\rangle \leq Z_{2 n}(V)$. Again, by Baer's theorem, $\gamma_{2 n+1}(V)$ has $(w, m)$-bounded order. Arguing as above, we may assume that $V$ is nilpotent of class at most $2 n$. We therefore remark that $a$ acts as a $(3 n)$-Engel automorphism on the set of $v$-values. Recall that $v(G)=H^{\prime}$ is nilpotent of $(w, m)$ bounded class. Applying Lemma 3.4 we obtain that $H^{\prime}\langle a\rangle$ is nilpotent of $(w, m)$ bounded class, where $a$ is any arbitrary commutator of $G$.

STEP 4. Assume that $H^{\prime}$ is a $p$-group for some prime $p$. Let $a$ be a commutator and $d$ be a $v$-value such that the $w$-value $\left[d,{ }_{n} a\right]$ is nontrivial.

Since $H^{\prime}\langle a\rangle$ is nilpotent, $a$ induces a $p$-automorphism of $H^{\prime}$. In other words, the image of $a$ in $G / C_{G}\left(H^{\prime}\right)$ has p-power order. By Lemma 3.2, the image of $a^{i}$ in $G / C_{G}\left(H^{\prime}\right)$ is a commutator whenever $i$ is not divisible by $p$. Therefore for each such $i$ there exists a commutator $a_{i}$ in $G$ such that $a^{i} \in a_{i} C_{G}\left(H^{\prime}\right)$. Thus, whenever $i$ is not divisible by $p$, we have the equality $\left[d,{ }_{n} a^{i}\right]=\left[d,{ }_{n} a_{i}\right]$ and hence $\left[d,{ }_{n} a^{i}\right]$ is a $w$-value.

Denote by $u=u(x, y)=[x, y, \ldots, y]$ the $(n-1)$-Engel word. Remark that $\left[d,{ }_{n} a\right]=\left[a^{-d},{ }_{n-1} a\right]^{a}$. Therefore $u\left(a^{-d}, a\right)$ is a $w$-value. More generally, $u\left(a^{-i d}, a^{i}\right)$ is a $w$-value for each $i$ not divisible by $p$. Let $K=\left\langle a^{d}, a\right\rangle$. Then the set

$$
\left\{u\left(a^{-i d}, a^{i}\right)^{x} \mid x \in K,(i, p)=1\right\}
$$

has at most $m$ elements. As $K \leq H^{\prime}\langle a\rangle$ is nilpotent of $(w, m)$-bounded class (see STEP 3), we deduce from Lemma 3.6 that the order of the element $\left[d,{ }_{n} a\right]$ is $(w, m)$ bounded.

We proved that the order of an arbitrary $w$-value $\left[d,{ }_{n} a\right]$ is bounded by a number which depends only on $w$ and $m$. Taking into account that $W$ is an abelian subgroup generated by $m$ such elements, we conclude that the order of $W$ is $(w, m)$-bounded. Thus, there exists a number $B=B(w, m)$ depending only on $w$ and $m$ such that if $H^{\prime}$ is a $p$-group, $W$ has order at most $B$. It is important that $B$ does not depend on $p$. 
STEP 5. We will now deal with the case where $H^{\prime}$ is not necessarily a $p$-group. Recall that $H^{\prime}$ is nilpotent. Let $p_{1}, p_{2} \ldots$ be the set of prime divisors of the order of $H^{\prime}$, and let $N_{i}$ denote the Hall $p_{i}{ }^{\prime}$-subgroup of $H^{\prime}$. We now know that the image of $W$ in $G / N_{i}$ has order at most $B$. It follows that $W$ embeds into a direct product of abelian groups of order at most $B$. Therefore the exponent of $W$ divides $B$ !. Thus $W$ is an abelian group with $m$ generators and exponent dividing $B$ !. We conclude that the order of $W$ is $(w, m)$-bounded. This completes the proof.

\section{References}

[1] A. Abdollahi, F.G. Russo, On a problem of P. Hall for Engel words, Arch. Math. (Basel) 97 (2011), 407-412.

[2] C. Acciarri, P. Shumyatsky, On words that are concise in residually finite groups. Journal of Pure and Applied Algebra 218 (2014) 130-134

[3] S. Black, Which words spell "almost nilpotent?" J. Algebra 221 (1999), 47-496.

[4] R.G. Burns, Y. Medvedev, Group laws implying virtual nilpotence. J. Aust. Math. Soc. 74 (2003), 295-312.

[5] E. Detomi, M. Morigi, P. Shumyatsky, Words of Engel type are concise in residually finite groups, Bull. Math. Sci. (2018). https://doi.org/10.1007/s13373-018-0130-4.

[6] J. D. Dixon, M. P. F. du Sautoy, A. Mann, D. Segal, Analytic pro- $p$ groups, London Math. Soc. Lecture Note Series 157, Cambridge Univ. Press., 1991.

[7] G. A. Fernández-Alcober, M. Morigi, Outer commutator words are uniformly concise. J. London Math. Soc. 82 (2010), 581-595.

[8] G. A. Fernández-Alcober, M. Morigi, G. Traustason, A note on conciseness of Engel words. Comm. Algebra 40 (2012), 257-2576.

[9] G. A. Fernández-Alcober, P. Shumyatsky, On bounded conciseness of words in residually finite groups. J. Algebra 500 (2018), 19-29.

[10] J.R.J Groves, Varieties of soluble groups and a dichotomy of P. Hall. Bull. Austral. Math. Soc. 5 (1971), 391-410,

[11] K. W. Gruenberg, Two theorems on Engel groups. Proc. Cambridge Philos. Soc. 49, (1953). $377-380$.

[12] K.W. Gruenberg, The Engel structure of linear groups. J. Algebra 3, 291-303 (1966).

[13] R. Guralnick, P. Shumyatsky, On rational and concise word. J. Algebra 429 (2015), 213-217.

[14] B. Huppert, Endliche Gruppen I, Springer, Berlin, 1967.

[15] B. Huppert, N. Blackburn, Finite Groups II, Springer Verlag, Berlin, 1982.

[16] M. Isaacs, Character Theory of Finite Groups, AMS, Providence, RI, 1976.

[17] S. V. Ivanov, P. Hall's conjecture on the finiteness of verbal subgroups. Izv. Vyssh. Ucheb. Zaved. 325 (1989), 60-70.

[18] A. Jaikin-Zapirain, On the verbal width of finitely generated pro-p groups. Rev. Mat. Iberoam. 168 (2008), 393-412.

[19] E. I. Khukhro and P. V. Shumyatsky, Bounding the exponent of a finite group with automorphisms. J. Algebra, 212 (1999), 363-374.

[20] M. Lazard, Sur les groupes nilpotents et les anneaux de Lie, Ann. Sci. École Norm. Supr. 71 (1954), 101-190.

[21] C. Martínez, E. Zelmanov, On Lie rings of torsion groups Bull. Math. Sci., 6 (2016), 371-377, DOI 10.1007/s13373-016-0085-2.

[22] N. Nikolov, D. Segal, On finitely generated profinite groups. I. Strong completeness and uniform bounds. Ann. Math. 165 (2007), 171-238.

[23] N. Nikolov, D. Segal, Powers in finite groups. Groups Geom. Dyn. 5 (2011), 501-507.

[24] A.Yu. Ol'shanskii, Geometry of Defining Relations in Groups, Math. Appl., vol. 70, Kluwer Academic Publishers, Dordrecht, 1991, Soviet Series.

[25] D.J.S. Robinson, Finiteness conditions and generalized soluble groups. Part 1. SpringerVerlag, New York-Berlin, 1972.

[26] D. Robinson, A Course in the Theory of Groups, Grad. Texts in Math., vol. 80, SpringerVerlag, New York, 1996.

[27] D. Segal, Words: notes on verbal width in groups. London Mathematical Society Lecture Note Series, 361. Cambridge University Press, Cambridge, 2009. 
[28] P. Shumyatsky, Applications of Lie ring methods to group theory, in " Nonassociative Algebra and Its Applications", (Eds R. Costa et al.), Marcel Dekker, New York, (2000), 373-395.

[29] J.S. Wilson and E. Zelmanov, Identities for Lie algebras of pro-p groups. J. Pure Appl. Algebra, 81 (1992), 103-109.

[30] E. Zelmanov, Nil Rings and Periodic Groups, The Korean Math. Soc. Lecture Notes in Math., Seoul, 1992.

[31] E. I. Zelmanov, Lie ring methods in the theory of nilpotent groups, in "Proc. Groups'93/St. Andrews, vol. 2", London Math. Soc. Lecture Note Ser. 212, Cambridge Univ. Press, 1995, $567-585$.

[32] E. I. Zelmanov, Lie algebras and torsion groups with identity. Journal of Combinatorial algebra, 1 (2017), 289-340.

Dipartimento di Matematica, Università di Padova, Via Trieste 63, 35121 Padova, ITALY

E-mail address: detomi@math.unipd.it

Dipartimento di Matematica, Università di Bologna, Piazza di Porta San Donato 5, 40126 Bologna, Italy

E-mail address: marta.morigi@unibo.it

Department of Mathematics, University of Brasilia, Brasilia-DF, 70910-900 Brazil E-mail address: pavel@unb.br 